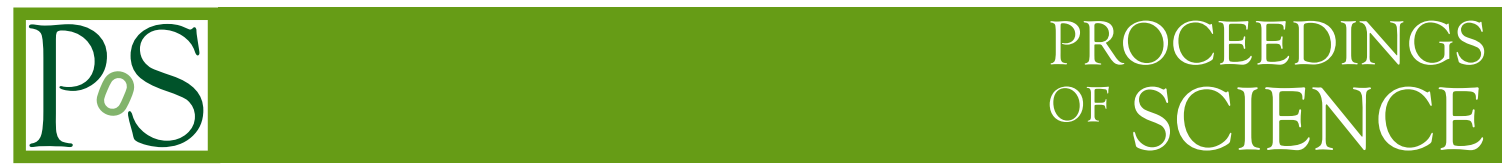

\title{
Jet and photon physics in pp collisions at the LHC
}

\author{
Vitaliano Ciulli * \\ Dipartimento di Fisica, Università di Firenze and Istituto Nazionale di Fisica Nucleare, Sezione \\ di Firenze, via G. Sansone 1, 50019 Sesto Fiorentino, Italy \\ E-mail: vitaliano.ciulliefi.infn.it
}

on behalf of the ALICE, ATLAS, CMS and LHCb collaborations

\begin{abstract}
Jets and photons production in proton-proton collisions at the Large Hadron Collider at CERN are key processes to test predictions of Quantum Chromodynamics and constraint the parton distribution functions of the proton. In this paper the most recent measurements from the LHC Collaborations are reviewed.
\end{abstract}

Fourth Annual Large Hadron Collider Physics

13-18 June 2016

Lund, Sweden

${ }^{*}$ Speaker. 


\section{Introduction}

Quantum Chromodynamics (QCD) is the fundamental theory describing strong interactions among partons, i.e. quarks and gluons. Jets and photons production in proton-proton collisions at the Large Hadron Collider (LHC) at CERN are key processes to test predictions of perturbative QCD (pQCD) over a wide region in phase space and constraint the parton distribution function (PDF) or the proton. In addition, they are among the main backgrounds to new physics searches and therefore needs to be estimated with the highest possible precision to allow discovering the tiniest deviations between data and theory.

The LHC collaborations, ALICE [1], ATLAS [2], CMS [3] and LHCb [4], performed many measurements of inclusive jet production, multi-jets production, jet properties and photon and diphoton production. In the following we will however review the most recent results, which include new measurements by ATLAS and CMS only.

\section{Jet physics}

Jets are generally reconstructed with the anti- $k_{\mathrm{t}}$ algorithm [5], with different values for the distance parameter $R$. ATLAS uses topological clusters [6] of cells in the calorimeter as input objects. In CMS and $\mathrm{LHCb}$, the particle-flow (PF) event algorithm [7, 8] first reconstructs and identifies each individual particle with an optimised combination of information from the various elements of the detector. Next, these PF objects are used as input to the jet reconstruction algorithm. ALICE uses tracks only or tracks plus energy deposits in the Electromagnetic Calorimeter to reconstruct jets in the regions $|\eta|<0.9$ and $|\eta|<0.7$, respectively [9]. The pileup (PU) of additional proton-proton interactions produce unwanted calorimetric energy depositions and additional tracks. Different strategies have been developed to subtract these effect depending on the jet recontruction. The main experimental difficulties for jet-based measurements are the jet energy calibration and resolution estimate and the subtraction of pileup effects.

The results are usually compared to fixed-order predictions at NLO precision, complemented with electroweak (EW) corrections, and to predictions of various Monte Carlo (MC) event generators that combine leading-order (LO) or next-to-leading-order (NLO) pQCD with the modeling of parton showers (PS), hadronisation (HAD) and multiparton interactions (MPI). In the case of fixed-order parton-level calculations, to be compared with measurement, they must be complemented with corrections for nonperturbative (NP) effects that involve the modeling of HAD and MPI, usually obtained from MC programs.

Measurements of inclusive jet production in proton-proton collisions have been performed at the LHC with data collected at different centre-of-mass energies, ranging from $2.76 \mathrm{GeV}$ to 13 $\mathrm{TeV}$. Using $13 \mathrm{TeV}$ data collected in 2015, the CMS experiment measured the double-differential inclusive jet cross section [10] as a function of the jet $p_{\mathrm{T}}$ and absolute jet rapidity $|y|$. The data samples correspond to an integrated luminosity of $71 \mathrm{pb}^{-1}$. The LHC operated with a $50 \mathrm{~ns}$ bunch spacing and the average number of pileup interactions observed is about 19. Figure 1 shows the double-differential inclusive jet cross section measurements, presented as a function of $p_{\mathrm{T}}$ for seven $|y|$ ranges, after unfolding for detector effects, using the anti- $k_{\mathrm{t}}$ algorithm with $R=0.7$ and 0.4 , respectively. The measurements are compared to the predictions from POWHEG [11] matched to 
PYTHIA8 [12] PS. The data are remarkably consistent with the predictions over a wide range of jet $p_{\mathrm{T}}$ from $114 \mathrm{GeV}$ up to $2 \mathrm{TeV}$. Results are also compared to the NLOJET++ [13] predictions using
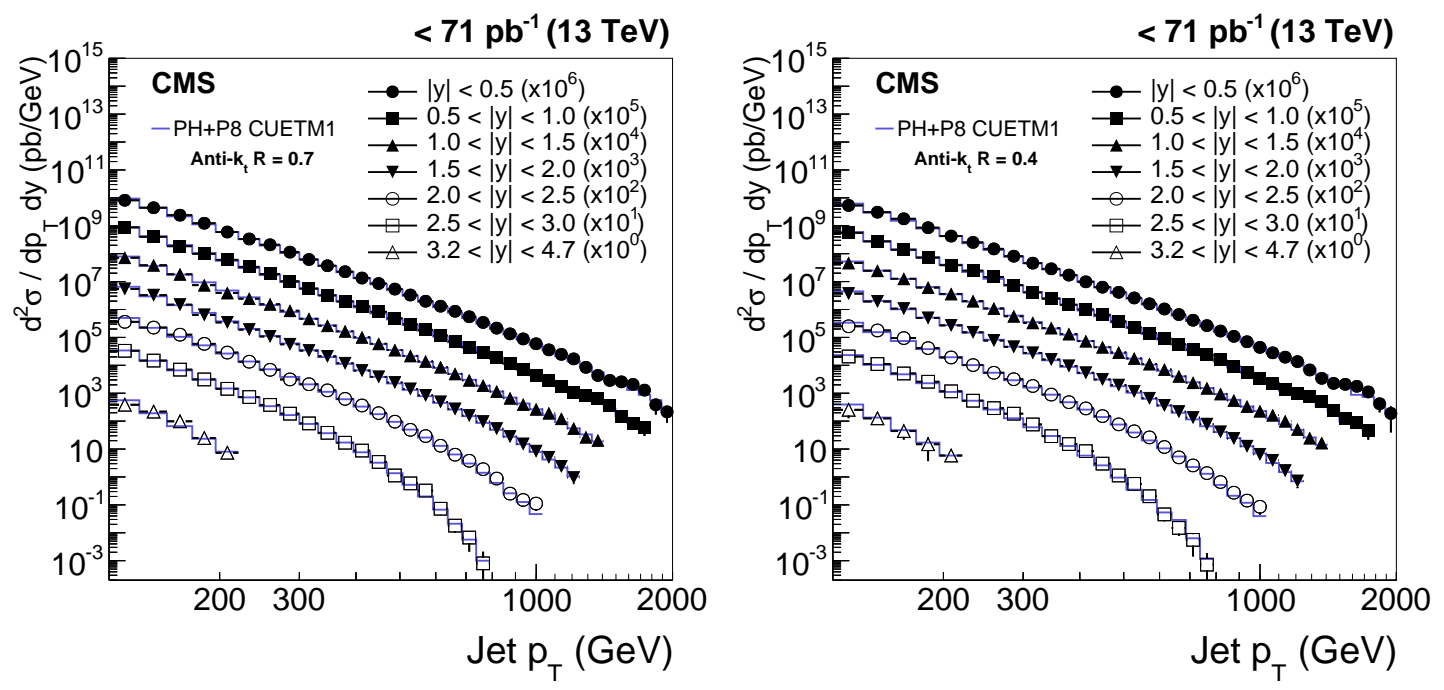

Figure 1: Double-differential inclusive jet cross section as function of jet $p_{\mathrm{T}}$ at $13 \mathrm{TeV}$ measured by CMS [10]. Data (points) and predictions from POWHEG + PYTHIA8 with tune CUETM1 (line) are shown. Jets are clustered with the anti- $k_{\mathrm{t}}$ algorithm with $R=0.7$ (left) and 0.4 (right).

the CT14 PDF set [14], corrected for NP and EW effects. The ratios of data over the NLOJET++ predictions are shown in Fig. 2 for the rapidity region $|y|<0.5$, but the same trend is observed in all the rapidity ranges. The relatively poor agreement for $R=0.4$ might be due to parton-shower and soft-gluon resummation contributions, which are missing in fixed-order calculations, or to higherorder effects.
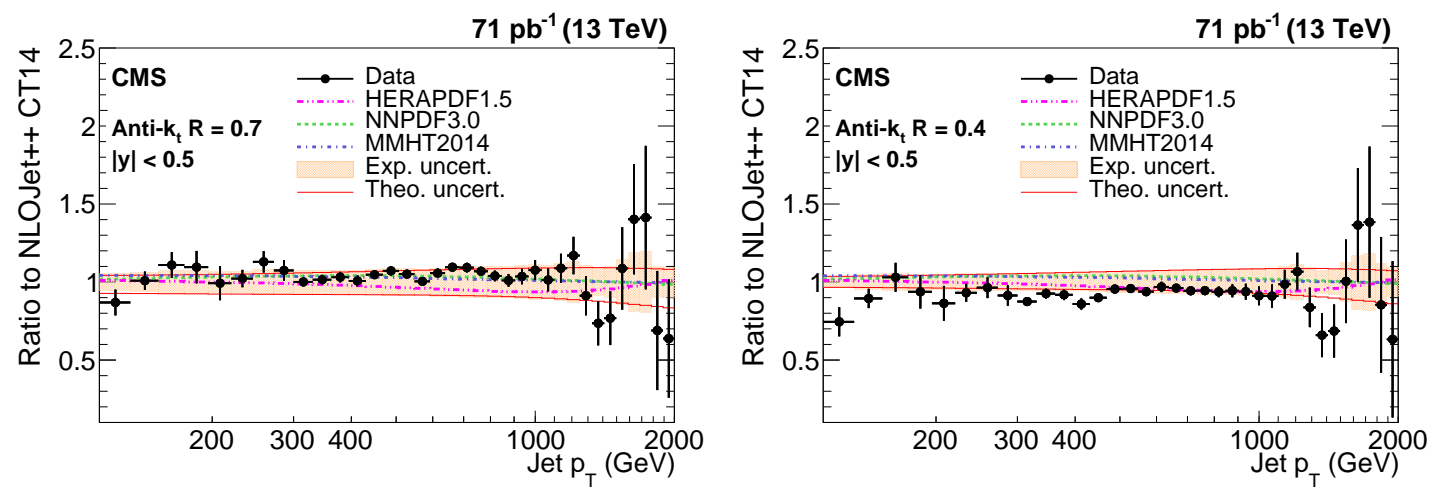

Figure 2: Ratio of values measured by CMS [10] to theoretical prediction from NLOJET++ using the CT14 PDF set and corrected for the NP and EW effects. Predictions employing three other PDF sets are also shown for comparison. Jets are clustered with the anti- $k_{\mathrm{t}}$ algorithm with a distance parameter of 0.7 (left) and 0.4 (right). The error bars correspond to the statistical uncertainties of the data and the shaded bands to the total experimental systematic uncertainties.

The CMS collaboration has also recently made available new results for the double-differential inclusive jet cross section at $8 \mathrm{TeV}$ [15] and $2.76 \mathrm{TeV}$ [16]. In the measurement at $8 \mathrm{TeV}$, the 
ratio to the cross section at $7 \mathrm{TeV}$ is also presented. A good agreement is observed in general between data and predictions, but for high transverse momentum some discrepancies are present, in particular in the region $1.0<|y|<1.5$, as shown in Figure 3. The inclusive jet cross section
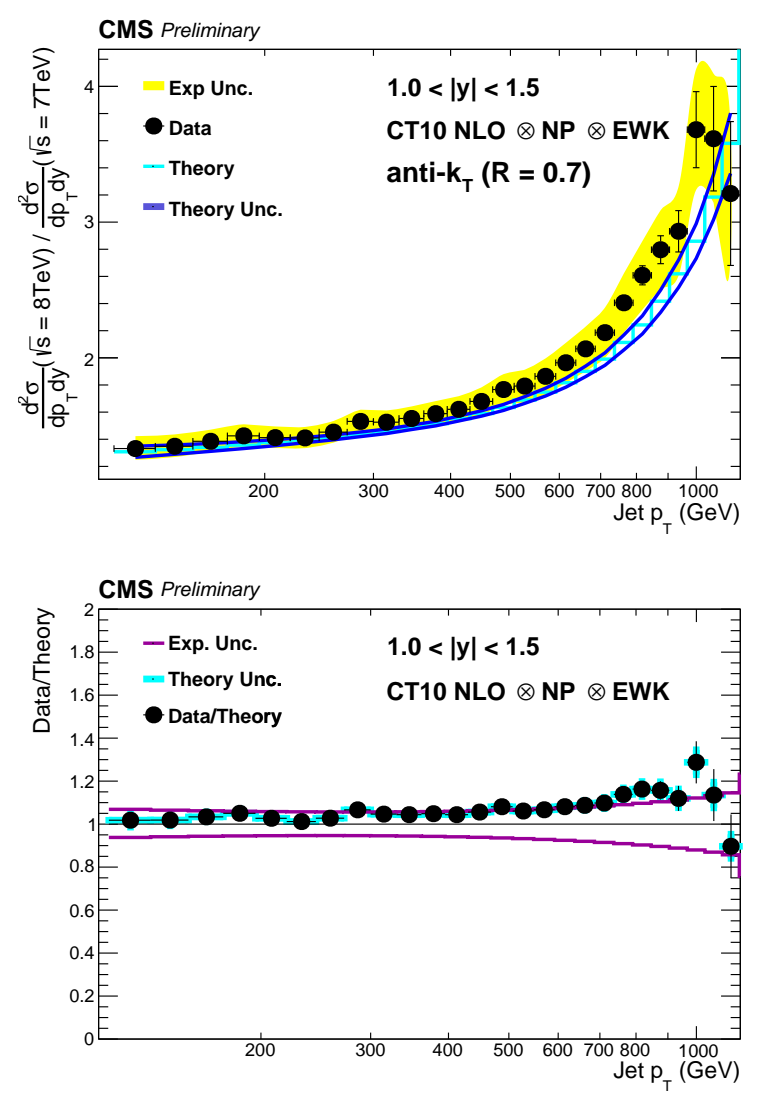

Figure 3: Ratio of double-differential inclusive jet cross sections between $\sqrt{\mathrm{s}}=8 \mathrm{TeV}$ and $\sqrt{\mathrm{s}}=7 \mathrm{TeV}$ for rapidity bins $1.0<|y|<1.5$ as measured by CMS [10]. In the top plots the yellow band shows the total experimental uncertainty, accounting for the correlation between different energies, and the theoretical prediction for the CT10 PDF set is overlaid. The bottom plot shows the ratio of the measured cross section ratio to its theoretical prediction, with the experimental uncertainty shown as a full line band, and theoretical uncertainties as a shaded band.

at $8 \mathrm{TeV}$ is very important for contraining the PDFs of the proton, in particular the gluon one, as it is shown in Figure 4, where the measurement is combined with the HERA data[17] using the HERAPDF method [18]. Furthermore the strong coupling constant $\alpha_{\mathrm{S}}$ is extracted from the measurement at $8 \mathrm{TeV}$, using for the calculation the CT10 NLO PDF set [19], which is found to provide the best agreement with data. With the entire probed $p_{\mathrm{T}}$ range and six different rapidity bins, the best fitted value is found to be $\alpha_{\mathrm{S}}\left(M_{\mathrm{Z}}\right)=0.1164_{-0.0043}^{+0.0060}$, which is compatible with the best current world average $\alpha_{\mathrm{S}}\left(M_{\mathrm{Z}}\right)=0.1185 \pm 0.0006$ [20]. Figure 5 shows that the running of $\alpha_{\mathrm{S}}(Q)$, measured for nine different values of renormalization scale between $86 \mathrm{GeV}$ and $1.5 \mathrm{TeV}$, is in good agreement with previous experiments and extends the measurement to the highest values of the renormalization scale.

The ATLAS Collaboration has recently measured the strong coupling constant using the trans- 


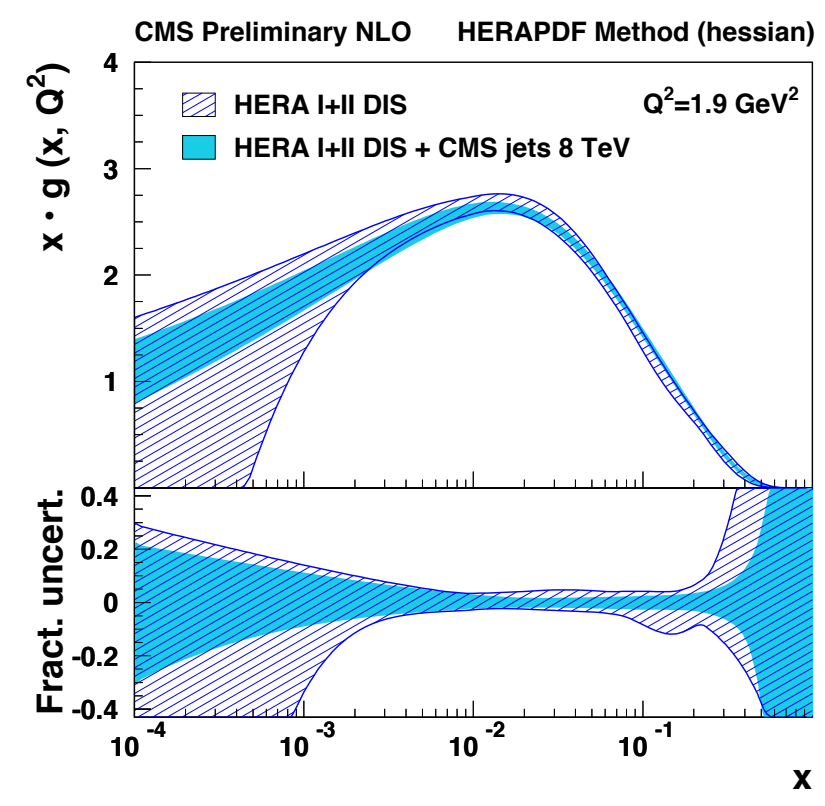

Figure 4: Distribution of gluon as a function of $x$ at the starting scale $Q^{2}=1.9 \mathrm{GeV}^{2}$, from [10]. The upper panel shows results of the fit to the HERA data and inclusive CMS jet measurements at $8 \mathrm{TeV}$ (shaded band), and to HERA only (hatched band), with their total uncertainties, as determined by using the HERAPDF method. In the bottom panels the fractional uncertainties are shown.

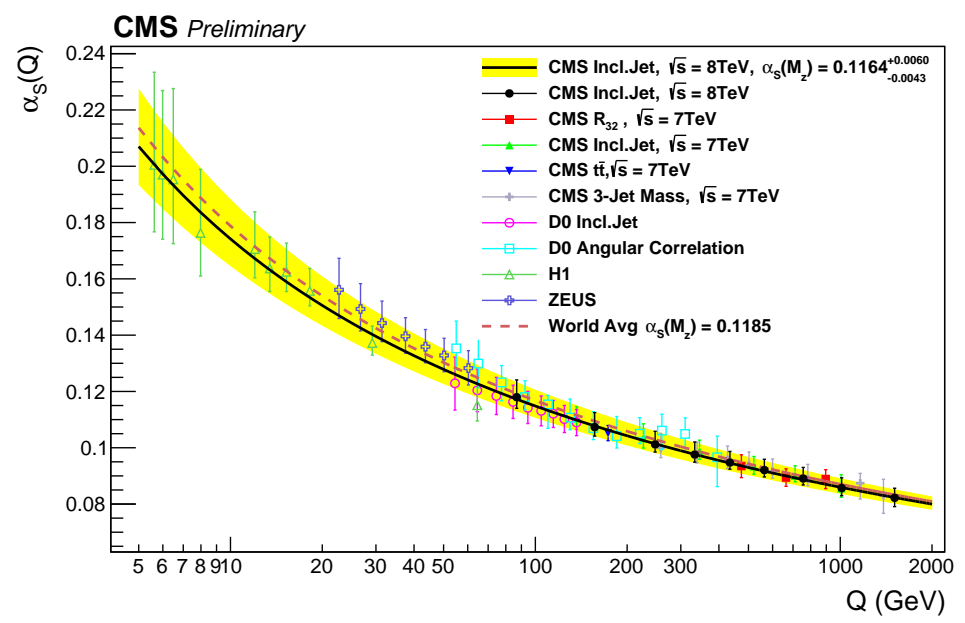

Figure 5: The running $\alpha_{\mathrm{S}}(Q)$ as a function of the scale $Q$, as obtained by using the CT10 NLO PDF set in [10]. The solid line and the uncertainty band are obtained by evolving the extracted $\alpha_{\mathrm{S}}\left(M_{\mathrm{Z}}\right)$ values by using the 2-loop 5-flavour renormalization group equations. The dashed line represents the evolution of the world average value. The black dots in the figure show the numbers obtained from the $\sqrt{s}=8 \mathrm{TeV}$ inclusive jet measurement by CMS. 


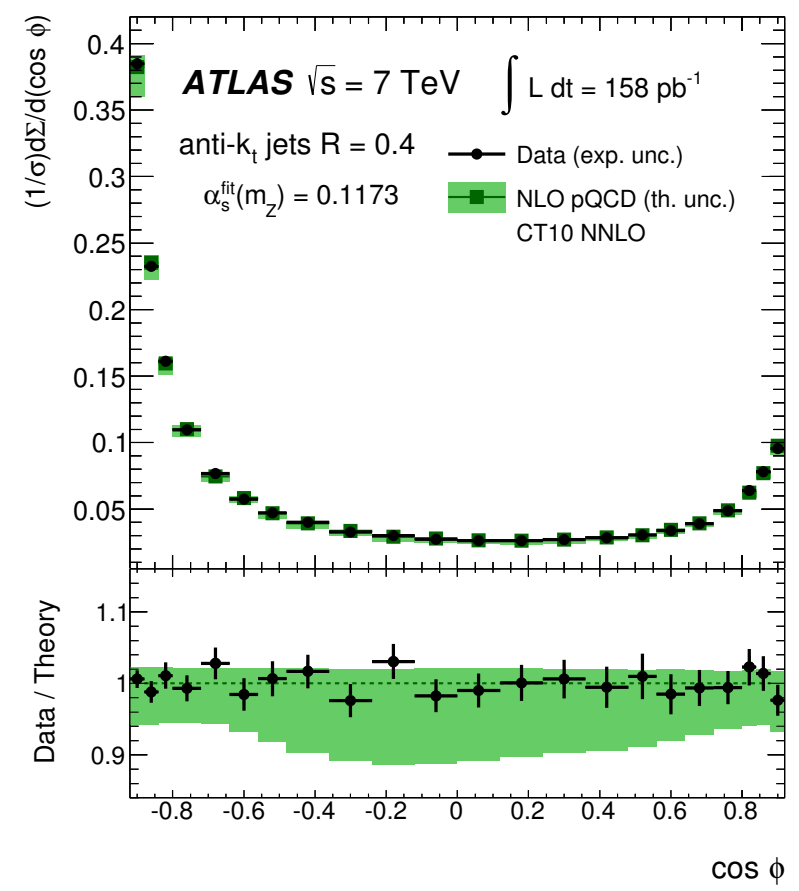

Figure 6: The unfolded distribution of the TEEC measured by ATLAS [21], compared with the results of a fit to pQCD NLO calculations including NP corrections. The green shaded band indicates the uncertainty on the theoretical predictions, which includes the sum in quadrature of uncertainties associated with scale, $\alpha_{\mathrm{S}}, \mathrm{PDF}$ and NP corrections.

verse energy-energy correlation (TEEC) function and its associated azimuthal asymmetry in multijet events at a centre-of-mass energy of $7 \mathrm{TeV}$ [21]. The average transverse momentum of the two leading jets is required to be larger than $250 \mathrm{GeV}$. The TEEC distribution is obtained from the $\cos \Delta \phi_{i j}$ between any pair of jets $i$ and $j$, weighted by

$$
w_{i j}=\frac{E_{T i} E_{T j}}{\left(\sum_{k} E_{T k}\right)^{2}},
$$

where $E_{T}$ is the jet transverse energy and the sum runs over all jets in the event. With respect to other observables the TEEC is less sensitive to the jet energy scale and resolution, and to PU. The measured TEEC distribution is shown in Figure 6, after unfolding the effects of the detector. In the same plot the result of a fit to pQCD NLO calculations from NLOJET++ including NP corrections is also shown. The measured value of $\alpha_{\mathrm{S}}\left(M_{\mathrm{Z}}\right)$ is

$$
\alpha_{\mathrm{S}}\left(M_{\mathrm{Z}}\right)=0.1173 \pm 0.0010(\text { exp. })_{-0.0020}^{+0.0063}(\text { scale }) \pm 0.0017(\mathrm{PDF}) \pm 0.0002(\mathrm{NP})
$$

and it is also in agreement with the world average. This value has been obtained with the CT10 NLO PDF set, which has the largest PDF uncertainty, covering the variations with different PDF sets.

The ATLAS Collaboration recently studied 4-jets events at $8 \mathrm{TeV}$ [22], exploring many variables sensitive to the topology and the different energy scales of the event. These observables have been compared to predictions from several MC generators and from fixed-order NLO calculations. 
The maximum rapidity difference among a jet pair, $\Delta y_{2 j}^{\max }$, shown in Figure 7, is one of the variables where some discrepancies are observed. The NLO predictions, BLACKHAT/SHERPA $[23,24]$ and
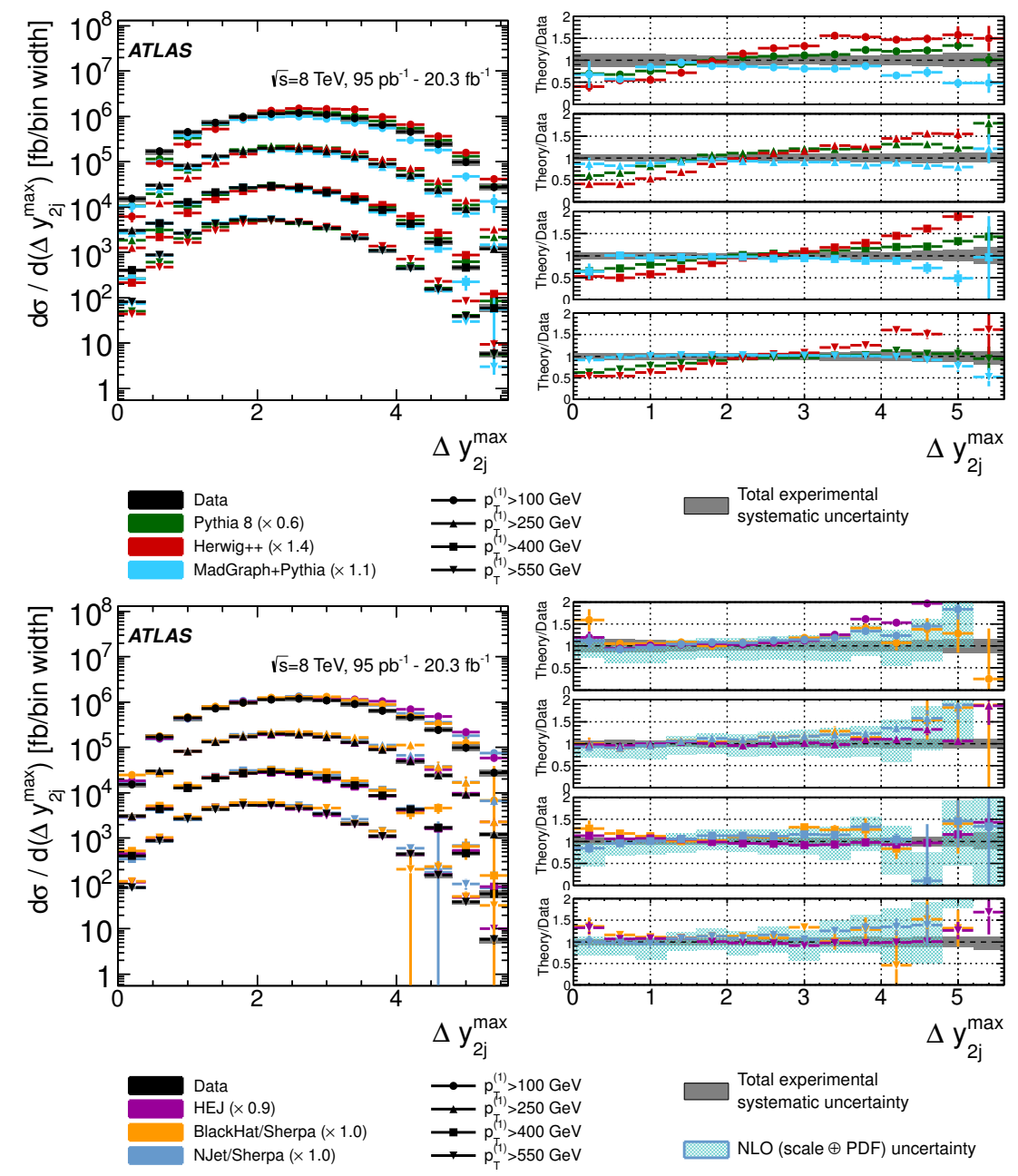

Figure 7: ATLAS unfolded four-jet differential cross section [22] as a function of $\Delta y_{2 j}^{\max }$, compared to different predictions: PYTHIA8, HERWIG++ and MADGRAPH +PYTHIA6 (top), and HEJ, NJET/SHERPA and BLACKHAT/SHERPA (bottom). The MC and HEJ predictions are multiplied by the factors indicated in the legend in order to be normalized to the events observed in the data. The left panel shows the full spectra and the right panel the ratios of the different predictions to the data. The solid band represents the total experimental systematic uncertainty. The patterned band represents the NLO scale and PDF uncertainties calculated from NJET/SHERPA. The scale uncertainties for HEJ (not drawn) typically range from -30\% to $+50 \%$. The ratio curves are formed by the central values and vertical uncertainty lines resulting from the propagation of the statistical uncertainties of the predictions and those of the unfolded data spectrum.

NJET/SHERPA [25, 26], are almost always compatible with the data within their theoretical uncertainties. The HEJ $[27,28]$ all-orders calculation and the multi-leg calculation with up to four partons in the matrix element (ME) generated by MADGRAPH [29] and matched to the PYTHIA6 [30] PS also provide a good description of the data. The $2 \rightarrow 2$ MC generators PYTHIA 8 and HERWIG++ [31] describe the data relatively poorly. These results show the need of more sophisticated calculations in many cases. 
The CMS Collaboration has also recently published new studies of the properties of multi-jets events at $7 \mathrm{TeV}[32,33]$ and $8 \mathrm{TeV}$ [34]. In Figure 8 the azimuthal decorrelation $\Delta \phi_{\text {dijet }}$ between the two jets with the largest transverse momenta at $8 \mathrm{TeV}$ is presented for seven regions of leading jet transverse momentum $p_{\mathrm{T}}^{\max }$ up to $2.2 \mathrm{TeV}$. The dijet azimuthal decorrelation is caused by the radiation of additional jets and probes the dynamics of multi-jet production. The results are compared to calculations in PQCD calculations from NLOJET++ with the CT10 NLO PDF set for 3-jet production with up to four outgoing partons. These fixed-order calculations provide NLO predictions for the range of $2 \pi / 3 \leq \Delta \phi_{\text {dijet }}<\pi$, corresponding to a final state with at least 3 jets, and LO predictions for $\pi / 2 \leq \Delta \phi_{\text {dijet }}<2 \pi / 3$, where at least 4 -jets must be present. The predictions are normalized separately in these two region and describe the data well over several order of magnitude, though they increasingly deviate from data for lower values of $\Delta \phi_{\text {dijet }}$, especially at low $p_{\mathrm{T}}^{\max }$. In Figure 9 the ratio of several MC generators predictions to the the data is shown. Similar

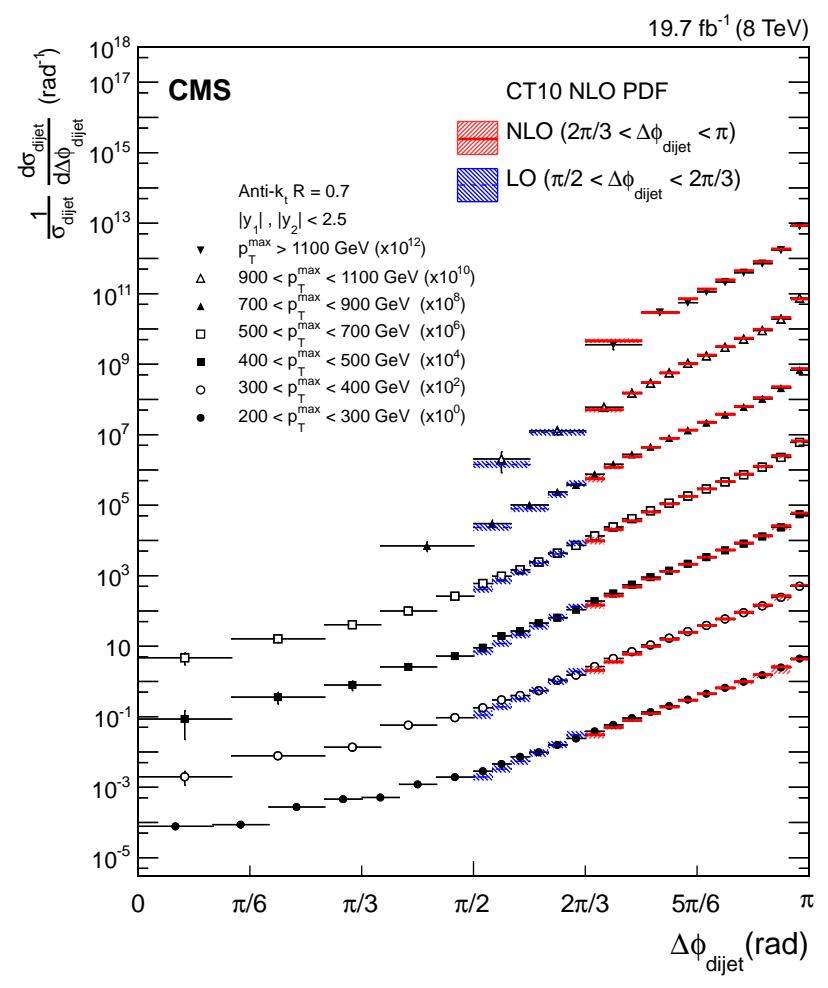

Figure 8: CMS normalized dijet cross section differential in $\Delta \phi_{\text {dijet }}$ for seven $p_{\mathrm{T}}^{\max }$ regions, scaled by multiplicative factors for presentation purposes [34]. The error bars on the data points include statistical and systematic uncertainties. Overlaid on the data (points) are predictions from LO (dashed line; $\pi / 2 \leq \Delta \phi_{\text {dijet }}<2 \pi / 3$ ) and NLO (solid line; $2 \pi / 3 \leq \Delta \phi_{\text {dijet }} \leq \pi$ ) calculations using the CT10 NLO PDF set.

conclusions as for the 4-jets analysis performed by ATLAS can be drawn. Event generators with only two outgoing partons at matrix-element fail to describe the data. This apply also to POWHEG that has NLO QCD corrections matched to the PYTHIA8 PS. In the case of MADGRAPH +PYTHIA6 from two to four outgoing partons are provided by the ME calculations and are matched to the PS, providing a very good description of the data down to very low $\Delta \phi_{\text {dijet }}$ values. 


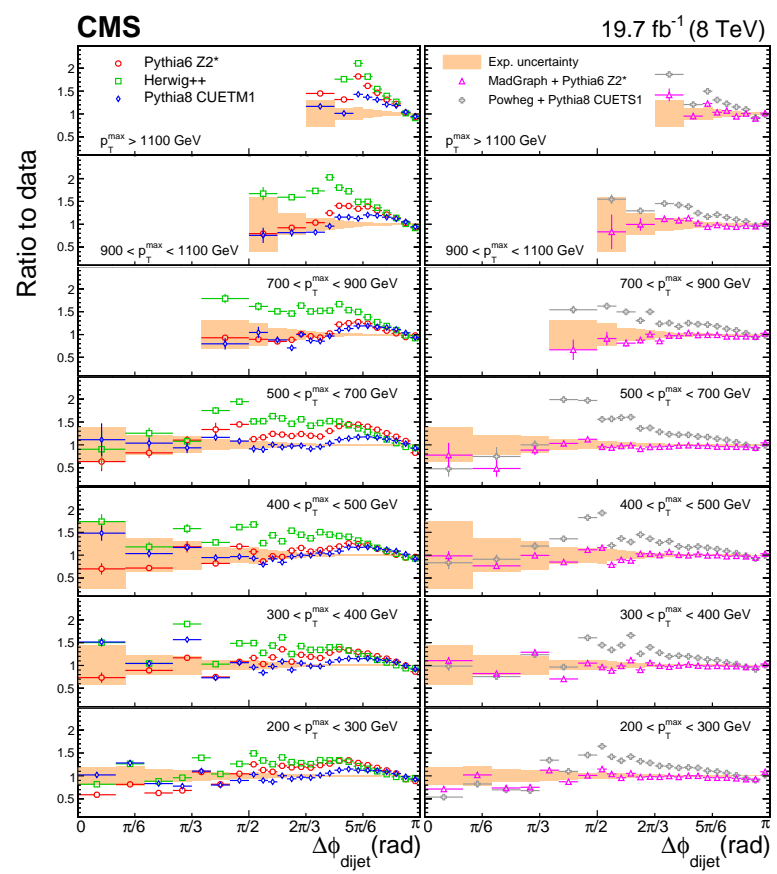

Figure 9: Ratios of PYTHIA6, HERWIG++, PYTHIA8, MADGRAPH + PYTHIA6, and POWHEG + PYTHIA8 predictions to the CMS normalized dijet cross section differential in $\Delta \phi_{\text {dijet }}$, for all $p_{\mathrm{T}}^{\max }$ regions [34]. The solid band indicates the total experimental uncertainty and the error bars on the MC points represent the statistical uncertainties of the simulated data.

Jets are not only a probe for pQCD or other high transverse momentum processes. They are also interesting for their properties, which depends mostly on quark fragmentation and hadronisation. An example of this kind of studies is the measurement of charged-particle multiplicity in jets $<n_{\text {charge }}>$ performed by ATLAS on the $8 \mathrm{TeV}$ data sample [35]. The multiplicity is measured including charged tracks with $p_{\mathrm{T}}>0.5,2$ or $5 \mathrm{GeV}$, for jets with $p_{\mathrm{T}}>50 \mathrm{GeV}$ and up to $1.5 \mathrm{TeV}$. Results are compared to HERWIG++, PYTHIA6 and PYTHIA8 with different underlying event tunings, showing that the description of data improves for the most recent tunes. The charged multiplicity is important to discriminate between quarks- and gluon-initiated jets, being larger in the case of a gluon jet. For this reason after unfolding the results for detector effects, the $\left\langle n_{\text {charge }}>\right.$ is extracted separately for quark and gluon jets as a function of the jet transverse momentum. Results are shown in Figure 10 and confirm that this value is larger for gluon jets, as expected, with a dependence on the jet transverse momentum.

The jet charge is another observable recently measured using $8 \mathrm{TeV}$ data by ATLAS [36] and CMS [37]. The jet charge is the $p_{\mathrm{T}}$ weighted sum of the charge of the particles in a jet. It is defined as

$$
Q^{\kappa}=\frac{1}{\left(p_{\mathrm{T}}\right)^{\kappa}} \sum_{i} Q_{i}\left(p_{\mathrm{T}}^{i}\right)^{\kappa}
$$

where the sum is over all particles $i$ in the jet with $p_{\mathrm{T}}>1 \mathrm{GeV}, Q_{i}$ is the charge of the particle, $p_{\mathrm{T}}^{i}$ is the particle transverse momentum, and $\kappa$ is a free parameter. The charge of the leading jet in dijets events is considered and different values of $\kappa$ are used: $0.3,0.5,0.7$ by ATLAS; 0.3, 0.6, 1.0 by 


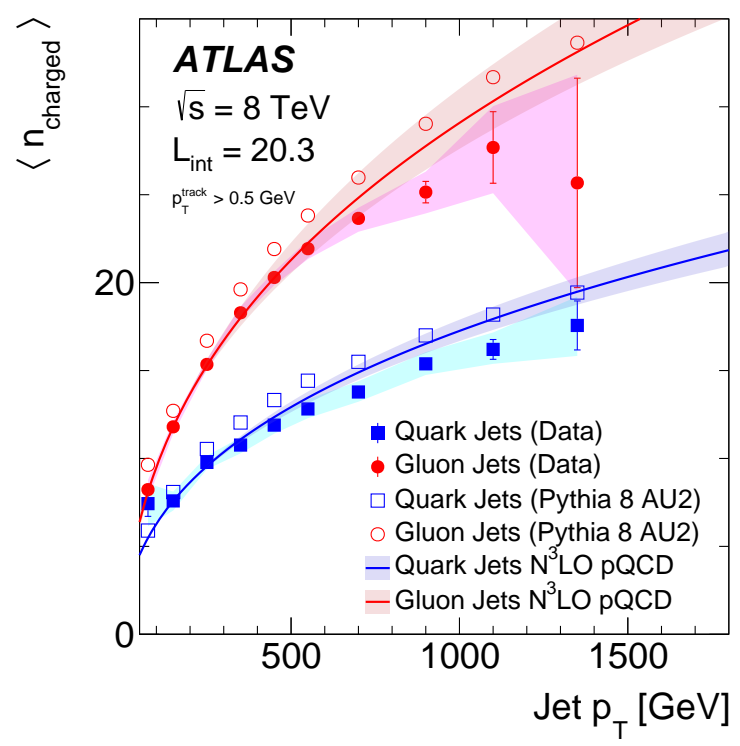

Figure 10: Jet $p_{\mathrm{T}}$ dependence of charged-particle multiplicity, for tracks $p_{\mathrm{T}}>0.5 \mathrm{GeV}$, for quark- and gluon-initiated jets, measured by ATLAS [35].

CMS. The CMS Collaboration used in addition two alternative definitions of the jet charge based on transverse and longitudinal momentum of the particles with respect to the jet axis also used. The full distribution of the jet charge is measured by CMS and unfolded for detector effects, as shown in Figure 11 for $\kappa=0.6$. The ATLAS collaboration measured instead the first and second moments
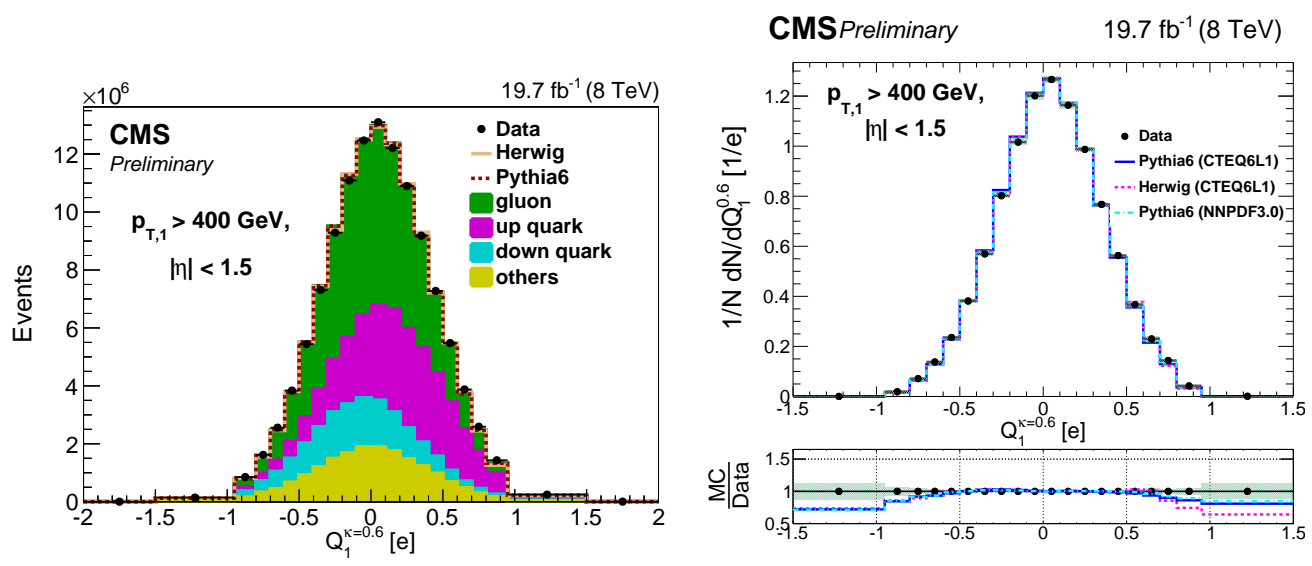

Figure 11: Distribution of the jet charge $Q^{\kappa}(\kappa=0.6)$ measured by CMS [37], compared to MC simulations. The left plot compare the sum of the contributions in PYTHIA6 and HERWIG++ to data (the flavor breakdown is carried out in PYTHIA6). The right plot compare the unfolded leading jet charge distribution with PYTHIA6 and HERWIG++ generators. Shaded uncertainty bands include both statistical and systematic effects, added in quadrature.

of the distributions. Results are shown in Figure 12 compared to MC predictions obtained using the CT10 NLO or the CTEQ6L1 [38] as the PDF set. The LO prediction agree with data within 
$5 \%$, while the NLO predictions are generally $10 \%$ below the data. There does not seem to be an effect from the NLO corrections in POWHEG. This observation is consistent with the expectation that the PDF and (nearly collinear) fragmentation are responsible for the jet charge distributions.
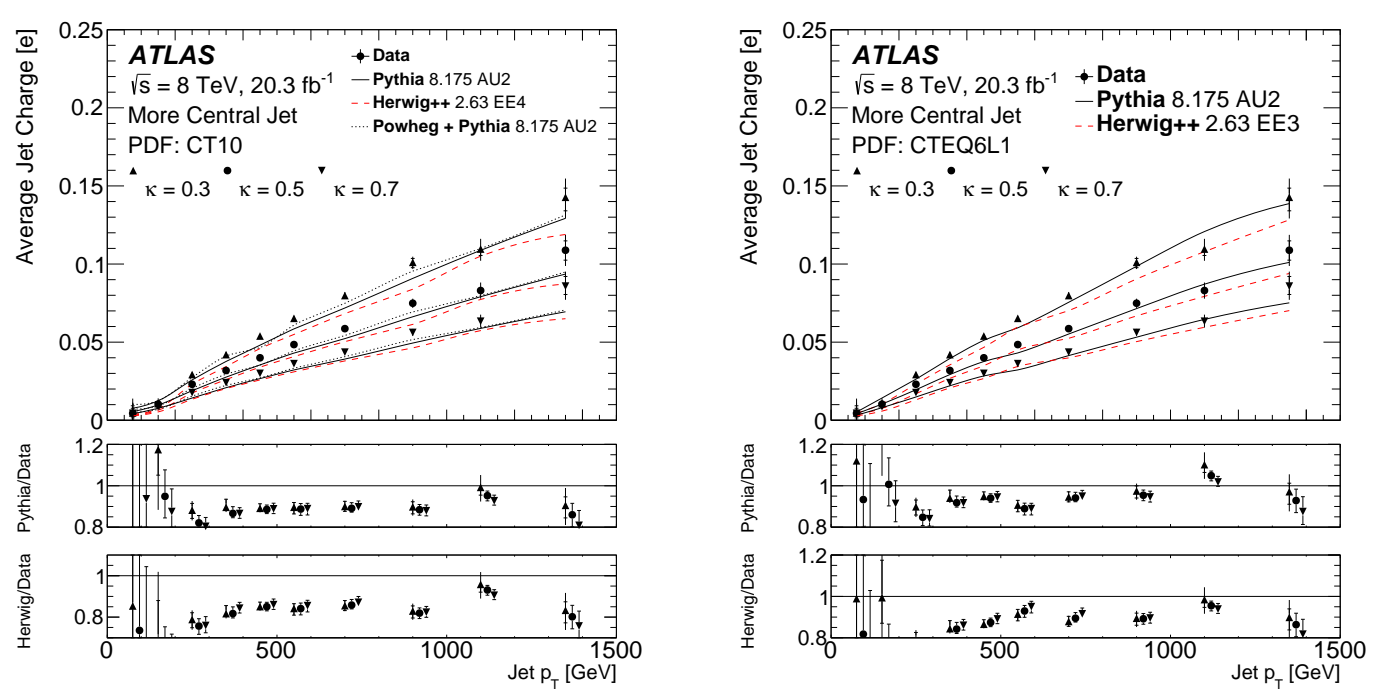

Figure 12: The average of the jet charge distribution measured by ATLAS [36] as a function of the jet $p_{\mathrm{T}}$ for $\kappa=0.3,0.5$, and 0.7 for the more central jet using CT10 (left) or CTEQ6L1 (right) as the PDF set. The crossed lines in the bars on the data indicate the systematic uncertainty and the full extent of the bars is the sum in quadrature of the statistical and systematic uncertainties.

\section{Prompt photon physics}

Prompt photons are those produced in the hard process, mainly through the LO process $q g \rightarrow$ $q \gamma$, and can therefore be used to study the gluon PDF. In addition, they are an important background to many interesting channel, e.g. Higgs decays into diphotons, and their production needs to be precisely measured. Furthermore, inclusive prompt photon production is made up of direct and fragmentation photons: direct photons are produced in the hard process, whereas fragmentation photons are generated in the parton fragmentation process. The dominant background to prompt photon production are photons originating from light neutral mesons decay $\left(\pi^{0}, \eta\right)$. The photon selection is designed to suppress the background from hadron decays, but also to reduce the fragmentation contribution, which is a less understood non-perturbative process. This is achieved by applying an isolation requirement.

The photon recontruction and identification starts from a cluster of energy deposits in the electromagnetic calorimeter (ECAL), supplemented by the informations in the tracker detector. If there are no tracks pointing to the cluster it is an unconverted photon candidate, while if there are two tracks coming from a conversion vertex or one track with no hits in the pixel detector it is a converted photon. Both candidates are kept for the following analyses, which are then tuned depending on the type.

In ATLAS photons are identified with a likelihood based on nine variables related to the lateral and longitudinal development of the shower in the LAr sampling ECAL [39]. In CMS the photon 
identification is based on the shape of the electromagnetic shower in the lead tungstate crystal ECAL [40]. Both experiments apply in addition an isolation requirement to further remove the background and the fragmentation photons as explained above. The identification with the shower shape and the isolation are partially uncorrelated and it is possible to extract from data the purity of the selected photon sample from the distribution in these two variables.

ATLAS recently published the inclusive prompt photon cross-section at $8 \mathrm{TeV}$ [41]. The measurement is performed for photons with an energy between $25 \mathrm{GeV}$ and $1.5 \mathrm{TeV}$, and for a pseudorapidity of up to $\eta=2.37$. Results are shown in Figure 13 together with NLO predictions from JetPhox [42]. Figure 14 shows the ratio of the theory predictions to the data: in addition

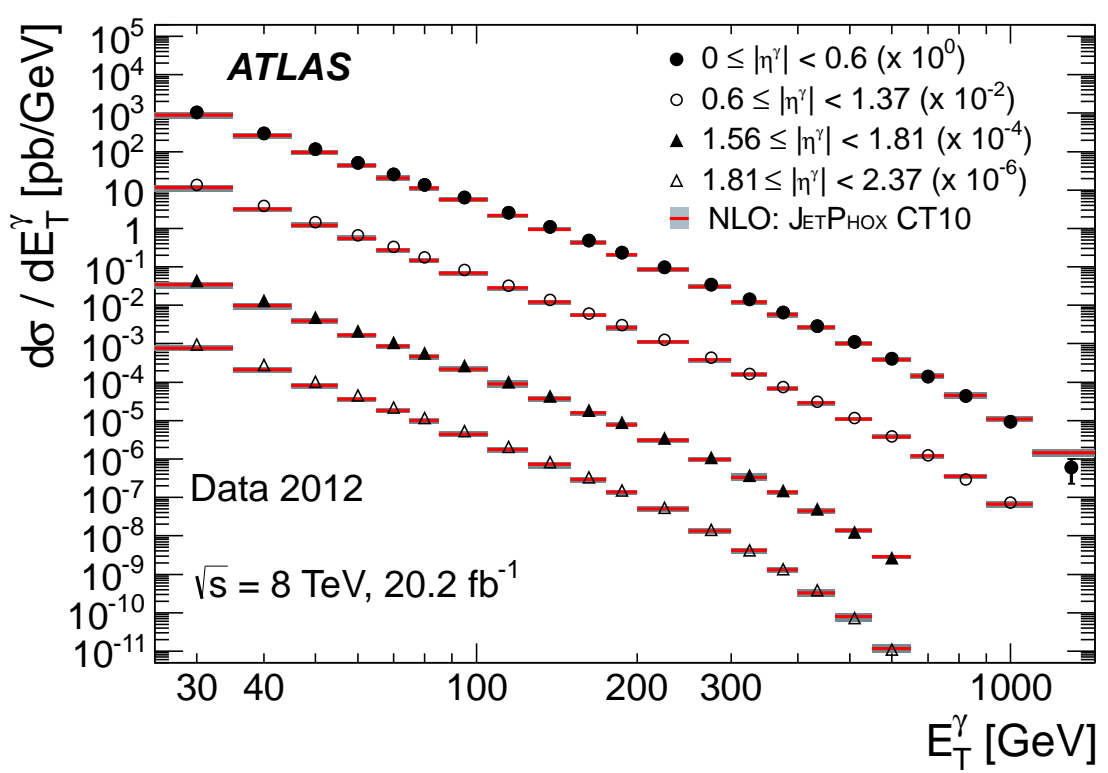

Figure 13: Differential cross sections of prompt photons in $8 \mathrm{TeV}$ data measured by ATLAS [41]. JETPHOX predictions are superimposed. The distributions are scaled by the specified factors to separate them visually.

to JetPhox, the MC programs PythiA and SHERPA are shown. Predictions from JetPhox with CT10 PDF set are 20\% lower than the data, though compatible within the uncertainty. Results from SHERPA, with CT10 PDF, and PYTHIA, with CTEQ6L1 PDF, show some significant deviations from the data: SHERPA is better for central photons, while PYTHIA is in good agreement with data for high $|\eta|$. In the case of SHERPA up to four partons can be present in the final state and the parton shower treats coherently gluon and photon emissions. That means that it is not possible to distinguish between direct and fragmentation photons. PYTHIA instead does the LO direct production in the ME and the fragmentation in the PS. As already noted, none of the two approaches is able to describe the data in the whole energy and pseudorapidity range.

The CMS Collaboration has measured the differential cross sections for the production of a photon pair in association with jets at $\sqrt{s}=7 \mathrm{TeV}$ [43]. The fraction of prompt diphoton events in data is extracted from a template fit to the photon isolation distribution. The signal template has been obtained from the data itself with the "random cone" technique, where the isolation energy is measured in a region separated from the photon. Several differential observables are studied 

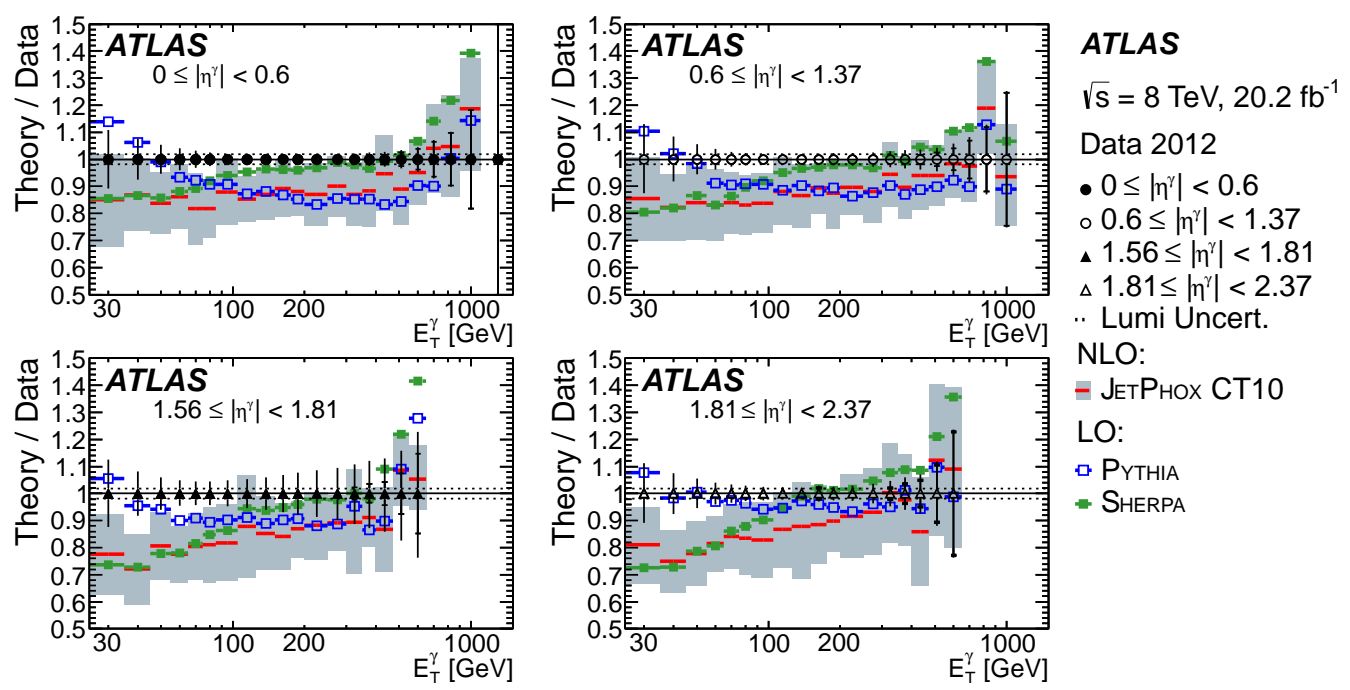

Figure 14: Ratio of theory (PYTHIA, SHERPA and JETPHOX) to ATLAS data for the differential prompt photon cross sections [41]. The statistical component of the uncertainty in the data is indicated by the horizontal tick marks whereas the whole error bar corresponds to the combined statistical and systematic uncertainty. The additional systematic uncertainty arising from the uncertainty in the integrated luminosity is displayed separately as a dotted line. The total uncertainty on JETPHOX calculations is displayed as a band.

with inclusive 1-jet and 2-jet selections and results are compared to LO and NLO QCD theoretical predictions from SHERPA, aMC @NLO [29], and GoSAM [44] event generators. As an example, the angular correlations between the leading jet and the photons are shown in Figure 15. The SHERPA and aMC@NLO predictions agree with the data for a large set of differential observables. The parton-level GoSAM prediction also describes the data well except for the angular correlations between photons and jets, where discrepancies are observed.

Finally, the ratio of the associated production of a $\mathrm{Z} / \gamma^{*}$ or a $\gamma$ with one or more jets, measured in proton-proton collisions at $8 \mathrm{TeV}$ center-of-mass energy by CMS [46], has been recently compared to new theoretical calculations in a study of the effect of EW correction [47]. In the limit of high transverse momentum of the vector boson the effects due to the mass of the $\mathrm{Z}$ boson are small, and the cross section ratio of $Z+j e t s$ to $\gamma+j$ ets is expected to become constant. Figure 16 compare the data to for events with a vector boson and at least one jet to fixed-order predictions from SHERPA +OPENLOOPS including both QCD and EW NLO corrections [48, 49]. The agreement of the combined NLO QCD+EW prediction with the CMS data is remarkable over the whole spectrum. At low transverse momentum NLO QCD corrections to the ratio are relevant due to mass effects, but sizable EW corrections (of different size for the two processes) alter the shape of the ratio prediction already much below $1 \mathrm{TeV}$. These results show the importance of combining NLO QCD and EW corrections in a unified framework.

\section{Conclusions}

We reviewed the most recent measurements of inclusive jet production, multi-jet production, 

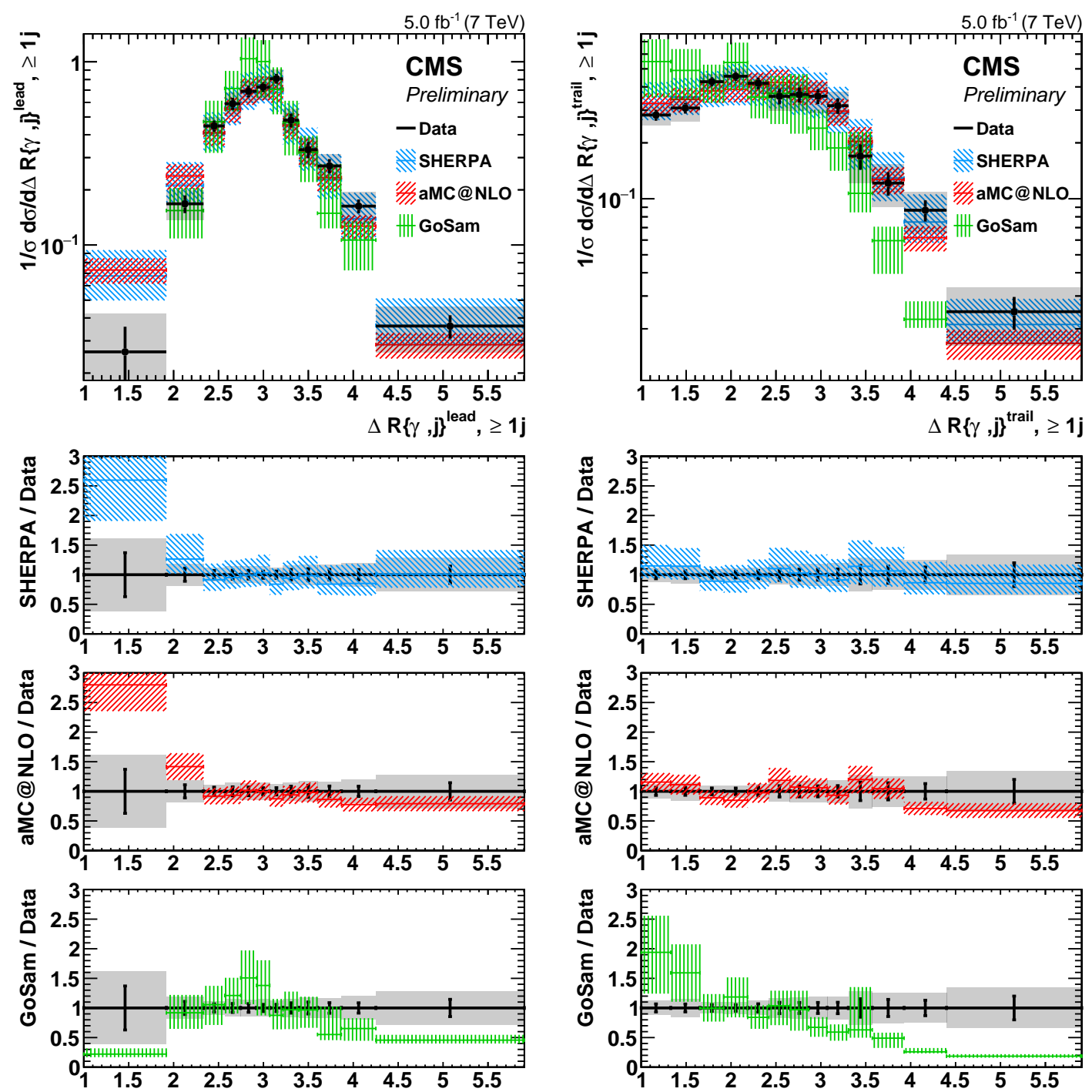

Figure 15: CMS diphoton differential cross section [43] as a function of the $\Delta R$ separation between the leading jet and the leading (left) or subleading (right) photon. All distributions are normalized to unitary area.

jet properties and photon and diphoton production in proton-proton collisions at the LHC. These measurements are important to test pQCD predictions and constraint the PDF of the proton. The results that have been presented show that event generators are generally in good agreement with the data. With the increase of the centre-of-mass energy to $13 \mathrm{TeV}$, however, there are hints that it is necessary to include higher order QCD and EW corrections to describe the data with the precision required by the high expected luminosity.

\section{References}

[1] ALICE Collaboration, The ALICE experiment at the CERN LHC, JINST 3 (2008) S08002. doi:10.1088/1748-0221/3/08/S08002 


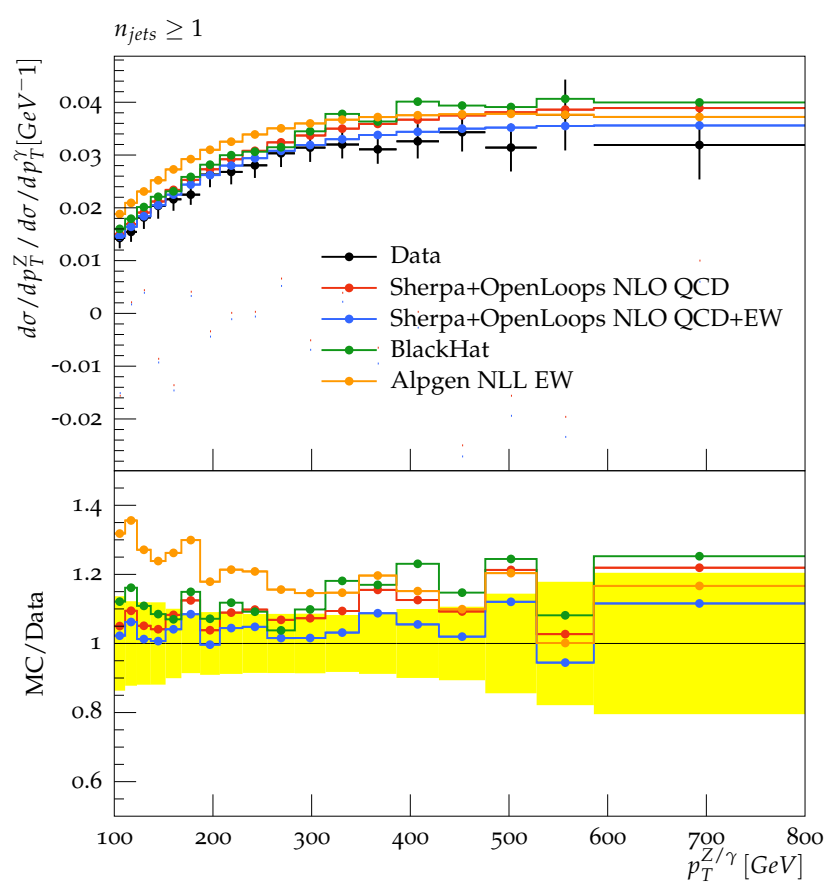

Figure 16: CMS measurement [46] of the ratio of $\mathrm{Z}+$ jets over $\gamma+$ jets at $8 \mathrm{TeV}$ pp center-of-mass energy, in events with at least 1 jet accompanying the boson. Superimposed are shown different predictions at NLO QCD, next-to-leading-logs (NLL) EW and NLO QCD+EW order, from [47]. For fixed order predictions the number of jets corresponds to the number of partons in the final state at the lowest order.

[2] ATLAS Collaboration, The ATLAS Experiment at the CERN Large Hadron Collider, JINST 3 (2008) S08003. doi:10.1088/1748-0221/3/08/S08003

[3] CMS Collaboration, The CMS experiment at the CERN LHC, JINST 3 (2008) S08004. doi:10.1088/1748-0221/3/08/S08004

[4] LHCb Collaboration, The LHCb Detector at the LHC, JINST 3 (2008) S08005. doi:10.1088/1748-0221/3/08/S08005

[5] M. Cacciari, G. P. Salam and G. Soyez, The Anti-k(t) jet clustering algorithm, JHEP 0804 (2008) 063, doi:10.1088/1126-6708/2008/04/063, [arXiv:0802.1189 [hep-ph]].

[6] W. Lampl et al., Calorimeter clustering algorithms: Description and performance, ATL-LARG-PUB-2008-002, ATL-COM-LARG-2008-003.

[7] CMS Collaboration, Particle-Flow Event Reconstruction in CMS and Performance for Jets, Taus, and MET, CMS-PAS-PFT-09-001.

[8] LHCb Collaboration, Study of forward Z + jet production in pp collisions at $\sqrt{s}=7 \mathrm{TeV}$, JHEP 1401 (2014) 033, doi:10.1007/JHEP01(2014)033, [arXiv:1310.8197 [hep-ex]].

[9] ALICE Collaboration, Measurement of the inclusive differential jet cross section in pp collisions at $\sqrt{s}=2.76 \mathrm{TeV}$, Phys. Lett. B 722 (2013) 262, doi:10.1016/j.physletb.2013.04.026, [arXiv:1301.3475 [nucl-ex]]. 
[10] CMS Collaboration, Measurement of the double-differential inclusive jet cross section in proton-proton collisions at sqrt(s) =13 TeV, [arXiv:1605.04436 [hep-ex]], Submitted to: Eur.Phys.J.C.

[11] S. Alioli, K. Hamilton, P. Nason, C. Oleari and E. Re, Jet pair production in POWHEG, JHEP 1104 (2011) 081, doi:10.1007/JHEP04(2011)081, [arXiv:1012.3380 [hep-ph]].

[12] T. Sjostrand, S. Mrenna and P. Z. Skands, A Brief Introduction to PYTHIA 8.1, Comput. Phys. Commun. 178 (2008) 852, doi:10.1016/j.cpc.2008.01.036, [arXiv:0710.3820 [hep-ph]].

[13] Z. Nagy, Next-to-leading order calculation of three jet observables in hadron hadron collision, Phys. Rev. D 68 (2003) 094002, doi:10.1103/PhysRevD.68.094002, [hep-ph/0307268].

[14] S. Dulat et al., New parton distribution functions from a global analysis of quantum chromodynamics, Phys. Rev. D 93 (2016) no.3, 033006, doi:10.1103/PhysRevD.93.033006, [arXiv:1506.07443 [hep-ph]].

[15] CMS Collaboration, Measurement of the double-differential inclusive jet cross section at sqrt $(s)=8$ $\mathrm{TeV}$, CMS-PAS-SMP-14-001.

[16] CMS Collaboration, Measurement of the inclusive jet cross section in pp collisions at $\sqrt{s}=2.76 \mathrm{TeV}$, Eur. Phys. J. C 76 (2016) no.5, 265, doi:10.1140/epjc/s10052-016-4083-z, [arXiv:1512.06212 [hep-ex]].

[17] H1 and ZEUS Collaborations, Combination of measurements of inclusive deep inelastic $e^{ \pm} p$ scattering cross sections and QCD analysis of HERA data, Eur. Phys. J. C 75 (2015) no.12, 580, doi:10.1140/epjc/s10052-015-3710-4, [arXiv:1506.06042 [hep-ex]].

[18] H1 and ZEUS Collaborations, Combined Measurement and QCD Analysis of the Inclusive e+- $p$ Scattering Cross Sections at HERA, JHEP 1001 (2010) 109, doi:10.1007/JHEP01(2010)109, [arXiv:0911.0884 [hep-ex]].

[19] H. L. Lai, M. Guzzi, J. Huston, Z. Li, P. M. Nadolsky, J. Pumplin and C.-P. Yuan, New parton distributions for collider physics, Phys. Rev. D 82 (2010) 074024, doi:10.1103/PhysRevD.82.074024, [arXiv:1007.2241 [hep-ph]].

[20] K. A. Olive et al. [Particle Data Group Collaboration], Review of Particle Physics, Chin. Phys. C 38 (2014) 090001. doi:10.1088/1674-1137/38/9/090001

[21] ATLAS Collaboration, Measurement of transverse energy-energy correlations in multi-jet events in pp collisions at $\sqrt{s}=7 \mathrm{TeV}$ using the ATLAS detector and determination of the strong coupling constant $\alpha_{\mathrm{s}}\left(m_{Z}\right)$, Phys. Lett. B 750 (2015) 427, doi:10.1016/j.physletb.2015.09.050, [arXiv:1508.01579 [hep-ex]].

[22] ATLAS Collaboration, Measurement of four-jet differential cross sections in $\sqrt{s}=8 \mathrm{TeV}$ proton-proton collisions using the ATLAS detector, JHEP 1512 (2015) 105, doi:10.1007/JHEP12(2015)105, [arXiv:1509.07335 [hep-ex]].

[23] C. F. Berger, Z. Bern, L. J. Dixon, F. Febres Cordero, D. Forde, H. Ita, D. A. Kosower and D. Maitre, An Automated Implementation of On-Shell Methods for One-Loop Amplitudes, Phys. Rev. D 78 (2008) 036003, doi:10.1103/PhysRevD.78.036003, [arXiv:0803.4180 [hep-ph]].

[24] Z. Bern et al., Four-Jet Production at the Large Hadron Collider at Next-to-Leading Order in QCD, Phys. Rev. Lett. 109 (2012) 042001, doi:10.1103/PhysRevLett.109.042001, [arXiv:1112.3940 [hep-ph]]. 
[25] S. Badger, B. Biedermann, P. Uwer and V. Yundin, Numerical evaluation of virtual corrections to multi-jet production in massless QCD, Comput. Phys. Commun. 184 (2013) 1981, doi:10.1016/j.cpc.2013.03.018, [arXiv:1209.0100 [hep-ph]].

[26] S. Badger, B. Biedermann, P. Uwer and V. Yundin, NLO QCD corrections to multi-jet production at the LHC with a centre-of-mass energy of $\sqrt{s}=8 \mathrm{TeV}$, Phys. Lett. B 718 (2013) 965, doi:10.1016/j.physletb.2012.11.029, [arXiv:1209.0098 [hep-ph]].

[27] J. R. Andersen and J. M. Smillie, Constructing All-Order Corrections to Multi-Jet Rates, JHEP 1001 (2010) 039, doi:10.1007/JHEP01(2010)039, [arXiv:0908.2786 [hep-ph]].

[28] J. R. Andersen and J. M. Smillie, Multiple Jets at the LHC with High Energy Jets, JHEP 1106 (2011) 010, doi:10.1007/JHEP06(2011)010, [arXiv:1101.5394 [hep-ph]].

[29] J. Alwall et al., The automated computation of tree-level and next-to-leading order differential cross sections, and their matching to parton shower simulations, JHEP 1407 (2014) 079, doi:10.1007/JHEP07(2014)079, [arXiv:1405.0301 [hep-ph]].

[30] T. Sjostrand, S. Mrenna and P. Z. Skands, PYTHIA 6.4 Physics and Manual, JHEP 0605 (2006) 026, doi:10.1088/1126-6708/2006/05/026, [hep-ph/0603175].

[31] M. Bahr et al., Herwig++ Physics and Manual, Eur. Phys. J. C 58 (2008) 639, doi:10.1140/epjc/s10052-008-0798-9, [arXiv:0803.0883 [hep-ph]].

[32] CMS Collaboration, Azimuthal decorrelation of jets widely separated in rapidity in pp collisions at $\sqrt{s}=7 \mathrm{TeV}$, Submitted to: JHEP, [arXiv:1601.06713 [hep-ex]].

[33] CMS Collaboration, Distributions of Topological Observables in Inclusive Three- and Four-Jet Events in pp Collisions at sqrt(s) = $7 \mathrm{TeV}$, Eur. Phys. J. C 75 (2015) no.7, 302, doi:10.1140/epjc/s10052-015-3491-9, [arXiv:1502.04785 [hep-ex]].

[34] CMS Collaboration, Measurement of dijet azimuthal decorrelation in pp collisions at $\sqrt{s}=8 \mathrm{TeV}$, Submitted to: Eur.Phys.J.C, [arXiv:1602.04384 [hep-ex]].

[35] ATLAS Collaboration, Measurement of the charged-particle multiplicity inside jets from $\sqrt{\mathrm{s}}=8 \mathrm{TeV}$ pp collisions with the ATLAS detector, Eur. Phys. J. C 76 (2016) no.6, 322, doi:10.1140/epjc/s10052-016-4126-5, [arXiv:1602.00988 [hep-ex]].

[36] ATLAS Collaboration, Measurement of jet charge in dijet events from $\sqrt{s}=8 \mathrm{TeV}$ pp collisions with the ATLAS detector, Phys. Rev. D 93 (2016) no.5, 052003, doi:10.1103/PhysRevD.93.052003, [arXiv:1509.05190 [hep-ex]].

[37] CMS Collaboration, Measurement of jet charge observables in dijet events at $\sqrt{s}=8 \mathrm{TeV}$, CMS-PAS-SMP-15-003.

[38] J. Pumplin, D. R. Stump, J. Huston, H. L. Lai, P. M. Nadolsky and W. K. Tung, New generation of parton distributions with uncertainties from global QCD analysis, JHEP 0207 (2002) 012, doi:10.1088/1126-6708/2002/07/012, [hep-ph/0201195].

[39] ATLAS Collaboration, Measurements of the photon identification efficiency with the ATLAS detector using 4.9 fbấL ک̌ 1 of pp collision data collected in 2011, ATLAS-CONF-2012-123.

[40] CMS Collaboration, Energy Calibration and Resolution of the CMS Electromagnetic Calorimeter in pp Collisions at $\sqrt{s}=7 \mathrm{TeV}$, JINST 8 (2013) P09009, [JINST 8 (2013) 9009],

doi:10.1088/1748-0221/8/09/P09009, [arXiv:1306.2016 [hep-ex]]. 
[41] ATLAS Collaboration, Measurement of the inclusive isolated prompt photon cross section in pp collisions at $\sqrt{s}=8 \mathrm{TeV}$ with the ATLAS detector, JHEP 1608 (2016) 005, doi:10.1007/JHEP08(2016)005, [arXiv:1605.03495 [hep-ex]].

[42] S. Catani, M. Fontannaz, J. P. Guillet and E. Pilon, Cross-section of isolated prompt photons in hadron hadron collisions, JHEP 0205 (2002) 028, doi:10.1088/1126-6708/2002/05/028, [hep-ph/0204023].

[43] CMS Collaboration, Production of pairs of isolated photons in association with jets in pp collisions at $\operatorname{sqrt}(s)=7 \mathrm{TeV}$, CMS-PAS-SMP-14-021.

[44] T. Gehrmann, N. Greiner and G. Heinrich, Photon isolation effects at NLO in $\gamma \gamma+$ jet final states in hadronic collisions, JHEP 1306 (2013) 058 Erratum: [JHEP 1406 (2014) 076], doi:10.1007/JHEP06(2014)076, 10.1007/JHEP06(2013)058, [arXiv:1303.0824 [hep-ph]].

[45] CMS Collaboration, Measurement of differential cross sections for the production of a pair of isolated photons in pp collisions at $\sqrt{s}=7 \mathrm{TeV}$, Eur. Phys. J. C 74 (2014) no.11, 3129, doi:10.1140/epjc/s10052-014-3129-3 [arXiv:1405.7225 [hep-ex]].

[46] CMS Collaboration, Comparison of the $Z / \gamma+$ jets to $\gamma+$ jets cross sections in pp collisions at $\sqrt{s}=8$ TeV, JHEP 1510 (2015) 128 Erratum: [JHEP 1604 (2016) 010], doi:10.1007/JHEP04(2016)010, 10.1007/JHEP10(2015)128 [arXiv:1505.06520 [hep-ex]].

[47] J. R. Andersen et al., Les Houches 2015: Physics at TeV Colliders Standard Model Working Group Report,”, pagg 61-77, arXiv:1605.04692 [hep-ph].

[48] S. Kallweit, J. M. Lindert, P. Maierhöfer, S. Pozzorini and M. Schönherr, NLO electroweak automation and precise predictions for W+multijet production at the LHC, JHEP 1504 (2015) 012, doi:10.1007/JHEP04(2015)012, [arXiv:1412.5157 [hep-ph]].

[49] S. Kallweit, J. M. Lindert, P. Maierhöfer, S. Pozzorini and M. Schönherr, NLO QCD+EW predictions for $V+$ jets including off-shell vector-boson decays and multijet merging, JHEP 1604 (2016) 021, doi:10.1007/JHEP04(2016)021, [arXiv:1511.08692 [hep-ph]]. 